Reducti on of I on Ther mal Diffusi vity Associ at ed with the Transiti on of the Radi al El ectri c Fi el d in Neut ral - Beam Heat ed Pl asmas in the Large Hel i cal Devi ce

\begin{tabular}{|l|l|}
\hline $\begin{array}{l}\text { j our nal or } \\
\text { publ i cat i on ti t l e }\end{array}$ & Physi cal Revi ew Let ter s \\
\hline vol une & Vol . 86 \\
\hline number & No. 23 \\
\hline page r ange & pp. 5297 - 5300 \\
\hline year & $2001-06-01$ \\
\hline URL & ht t p: //hdl . handl e. net /10655/103 \\
\hline
\end{tabular}




\title{
Reduction of Ion Thermal Diffusivity Associated with the Transition of the Radial Electric Field in Neutral-Beam-Heated Plasmas in the Large Helical Device
}

\author{
K. Ida, H. Funaba, S. Kado,* K. Narihara, K. Tanaka, Y. Takeiri, Y. Nakamura, N. Ohyabu, K. Yamazaki, \\ M. Yokoyama, S. Murakami, N. Ashikawa, P. C. deVries, ${ }^{\dagger}$ M. Emoto, M. Goto, H. Idei, K. Ikeda, S. Inagaki, \\ N. Inoue, M. Isobe, K. Itoh, O. Kaneko, K. Kawahata, K. Khlopenkov, A. Komori, S. Kubo, R. Kumazawa, Y. Liang, ${ }^{\ddagger}$ \\ S. Masuzaki, T. Minami, J. Miyazawa, T. Morisaki, S. Morita, T. Mutoh, S. Muto, Y. Nagayama, H. Nakanishi, \\ K. Nishimura, N. Noda, T. Notake, ${ }^{\S}$ T. Kobuchi, S. Ohdachi, K. Ohkubo, Y. Oka, M. Osakabe, T. Ozaki, \\ R. O. Pavlichenko, B. J. Peterson, A. Sagara, K. Saito, ${ }^{\S}$ S. Sakakibara, R. Sakamoto, H. Sanuki, H. Sasao, M. Sasao, \\ K. Sato, M. Sato, T. Seki, T. Shimozuma, M. Shoji, H. Suzuki, S. Sudo, N. Tamura ${ }^{\ddagger}$ K. Toi, T. Tokuzawa, Y. Torii, ${ }^{\S}$ \\ K. Tsumori, T. Yamamoto, H. Yamada, I. Yamada, S. Yamaguchi, S. Yamamoto, ${ }^{\S}$ Y. Yoshimura, K. Y. Watanabe, \\ T. Watari, Y. Hamada, O. Motojima, and M. Fujiwara \\ National Institute for Fusion Science, 322-6 Oroshi-cho, Toki, 509-5292, Japan
}

(Received 4 January 2001)

\begin{abstract}
Recent large helical device experiments revealed that the transition from ion root to electron root occurred for the first time in neutral-beam-heated discharges, where no nonthermal electrons exist. The measured values of the radial electric field were found to be in qualitative agreement with those estimated by neoclassical theory. A clear reduction of ion thermal diffusivity was observed after the mode transition from ion root to electron root as predicted by neoclassical theory when the neoclassical ion loss is more dominant than the anomalous ion loss.
\end{abstract}

DOI: 10.1103/PhysRevLett.86.5297

Neoclassical ion transport is important in stellarator plasmas, because the helical ripple losses are comparable to or sometimes even higher than the anomalous losses in contrast to those in tokamaks. The crucial issues of neoclassical ion transport are (1) the reduction of ion thermal diffusivity due to a large positive radial electric field in the electron root [1-3], and (2) the reduction of ion thermal diffusivity due to the optimization of the magnetic field structure ( $\sigma$ optimization) [3-6]. However, there has been no experimental study to test these issues on the neoclassical ion transport in stellarator plasmas. This is because the transition of the radial electric field from small negative (ion root) to large positive (electron root) was observed only in plasmas with the assistance of electron cyclotron heating $(\mathrm{ECH})$, where electron heating is dominant [7-11]. The ion temperature is much lower than the electron temperature because ions are heated only by the energy exchange between ions and electrons. In these experiments, the significant increase of electron temperature and a clear reduction of electron thermal diffusivity were observed in the plasma core in the electron root. However, no reduction of ion thermal diffusivity was observed because of the lack of direct ion heating. There have been no experimental results to show the improvement of ion transport in the electron root, although a significant improvement of ion transport (rather than the electron transport) is predicted by the neoclassical theory [6]. This paper describes the experimental results of the neoclassical feature of ion transport for the first time, the reduction of ion thermal diffusivity due to the transition to the large positive electric field (electron root), and/or the optimization of the magnetic field structure ( $\sigma$ optimization).
PACS numbers: 52.55.-s, 52.50.Gj

The large helical device (LHD) [12] is a Heliotron device (poloidal period number $L=2$, and toroidal period number $M=10$ ) with a major radius of $R_{\mathrm{ax}}=3.5-4.1 \mathrm{~m}$, an average minor radius of $0.6 \mathrm{~m}$, and magnetic field up to $3 \mathrm{~T}$. The radial electric field $\left(E_{r}\right)$ is derived from the poloidal and toroidal rotation velocity and pressure gradient of neon impurity measured with charge exchange spectroscopy [13] at the midplane in LHD (vertically elongated cross section) using a radial force balance. The radial force balance equation can be expressed as $E_{r}=\left(e n_{I} Z_{I}\right)^{-1}\left(\partial p_{I} / \partial r\right)-\left(v_{\theta} B_{\phi}-v_{\phi} B_{\theta}\right)$, where $B_{\phi}$ and $B_{\theta}$ are toroidal and poloidal magnetic field, and $Z_{I}, n_{I}$, and $p_{I}$ are ion charge, density, and pressure of impurity measured, respectively.

In the LHD, the transition from negative electric field (ion root) to positive electric field (electron root) is observed for the first time in plasmas with neutral beam injection (NBI) heating alone at a low density of $(0.4-1.0) \times$ $10^{19} \mathrm{~m}^{-3}$. In previous experiments [7-11], the transition from ion root to electron root was observed only in plasmas with $\mathrm{ECH}$, therefore it has been also open to question as to whether the nonthermal electrons driven by $\mathrm{ECH}$ are required to achieve the transition. The transition of radial electric field from ion root to electron root clearly demonstrates that nonthermal electrons are not necessary for the transition, because there are no nonthermal electrons in NBI heated plasma. Figure 1 shows the density dependence of the radial electric field near the plasma edge ( $\rho=$ $0.9)$ for the standard configuration $\left[R_{\mathrm{ax}}=3.75 \mathrm{~m}\right]$ and the inward shifted configuration $\left[R_{\mathrm{ax}}=3.6 \mathrm{~m}\right]$. By shifting the magnetic axis inward, the magnetic field structure is optimized for neoclassical transport, and the loss cone 

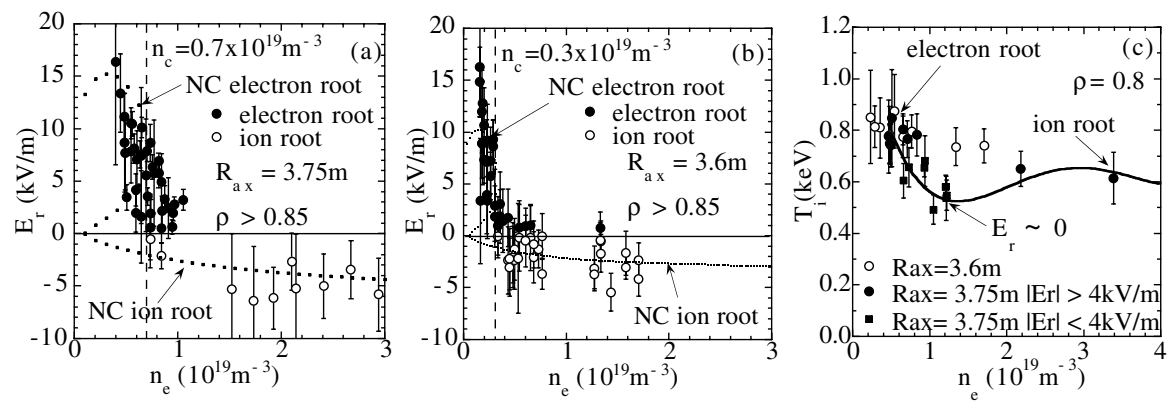

FIG. 1. Density dependence of radial electric field at $\rho>0.85$ for the plasmas with (a) standard configuration $\left(R_{\mathrm{ax}}=3.75 \mathrm{~m}\right)$ and (b) inward shifted configuration $\left(R_{\mathrm{ax}}=3.6 \mathrm{~m}\right)$. The dashed lines in (a) and (b) are neoclassical predictions. (c) Ion temperature at $\rho=0.8$ as a function of electron density for these plasmas. The solid line is a guide for the eye for the ion temperature in the standard configuration.

regime is expected to be significantly reduced $[3,6]$. The inward shift of magnetic axis in LHD is equivalent to the $\sigma$ optimization due to the pitch modulation. As we discuss later, the neoclassical transport loss is predicted to be large enough to exceed the anomalous loss for the configuration with the magnetic axis of $3.75 \mathrm{~m}$, while it is relatively small for that of $3.6 \mathrm{~m}$. The transition of electron root to ion root is observed at $0.7 \times 10^{19} \mathrm{~m}^{-3}\left(T_{e}=\right.$ $0.6 \mathrm{keV}$ at $\rho=0.9$ ) for the plasma with $R_{\mathrm{ax}}=3.75 \mathrm{~m}$, while it is observed at the lower electron density $0.3 \times$ $10^{19} \mathrm{~m}^{-3}\left(T_{e}=0.7 \mathrm{keV}\right.$ at $\left.\rho=0.9\right)$ for the plasma with $R_{\text {ax }}=3.75 \mathrm{~m}$, while it is observed at the lower electron density $0.3 \times 10^{19} \mathrm{~m}^{-3}\left(T_{e}=0.7 \mathrm{keV}\right.$ at $\left.\rho=0.9\right)$ for the plasma with $R_{\mathrm{ax}}=3.6 \mathrm{~m}$, where the plasma is well into the collisionless regime $\left(\nu_{e}^{*}<0.1\right)$. The difference of transition density is due to the differences in the ratio of edge ion temperature to electron temperature. The ion temperature, $T_{i}$, is 0.5 to 0.9 times the electron temperature, $T_{e}$ at $\rho=0.9$ for the plasma with $R_{\mathrm{ax}}=3.75 \mathrm{~m}$, while $T_{i}=T_{e}$ for the plasma with $R_{\mathrm{ax}}=3.6 \mathrm{~m}$.

The edge radial electric field sharply increases up to $15 \mathrm{kV} / \mathrm{m}$ in the electron root as the electron density is decreased, while the absolute values increase gradually up to $-5 \mathrm{kV} / \mathrm{m}$ in the ion root over a wide range of electron density of $(1.0-3.0) \times 10^{19} \mathrm{~m}^{-3}$. The transition from ion root (negative $E_{r}$ ) to electron root (positive $E_{r}$ ) is observed at $\rho>0.85$, and there is no large radial electric field observed in the plasma core. The behavior of the radial electric field measured can be explained by neoclassical theory [2] as shown in Fig. 1. The absolute values of radial electric field measured in the electron or ion root are the levels predicted by neoclassical theory. The critical electron density required for transition from electron root to ion root has good agreement with the neoclassical prediction. The electric field is negative when the electron density is above $1.0 \times 10^{19} \mathrm{~m}^{-3}$, and it becomes more negative as the electron density gradients are increased. The radial electric field in the ion root is typically small, and the absolute values are less than $5 \mathrm{kV} / \mathrm{m}$ for most discharges (see Fig. 1).

Figure 1(c) shows the density dependence of ion temperature near the plasma edge $(\rho=0.8)$. The edge ion temperature for the plasma with $R_{\mathrm{ax}}=3.75 \mathrm{~m}$ gradually increases as the electron density is decreased in the ion root $\left(n_{e}>2 \times 10^{19} \mathrm{~m}^{-3}\right)$. However, the ion temperature starts to decrease at the electron density where the radial electric field is nearly zero $\left(-4<E_{r}<4 \mathrm{kV} / \mathrm{m}\right)$. The ion temperature recovers and increases sharply as the electron density decreases in the electron root $\left(n_{e}<\right.$ $\left.0.7 \times 10^{19} \mathrm{~m}^{-3}\right)$. On the other hand, the electron temperature monotonically increases as the electron density is decreased. The drop of temperature near $E_{r}=0$ is observed only in the ions. This result shows that the neoclassical heat flux increases as the radial electric field becomes small. However, the neoclassical heat flux does not become so large as to prevent the access to the electron root. If the neoclassical heat flux at the zero radial electric field is large, the collisionality cannot be small enough to make the transition from ion root to electron root, unless the heating power is significantly increased. This behavior is observed in the standard configuration where the neoclassical heat flux is expected to exceed the anomalous heat flux. On the contrary, the similar behavior is not observed in the inward shifted configuration, in which the neoclassical heat flux is expected to be small compared to the anomalous transport. It should be noted that the transition from ion root to electron root takes place even if the neoclassical contribution is not dominant in the heat flux. This is due to the fact that the neoclassical bipolar flux is dominant and the anomalous bipolar flux is small if it exists, although the anomalous heat flux is dominant for the inward shifted plasma $\left(R_{\mathrm{ax}}=3.6 \mathrm{~m}\right)$.

Figure 2 shows the radial profiles of electron density and temperature and ion temperature and radial electric field. The density profiles in LHD are flat or slightly hollow. The electron density is low enough to decouple the ion transport from electron transport, because the energy exchange due to the collisions between ions and electrons are negligible. The temperature shows an edge pedestal [14] at $\rho>0.9$, but there is no clear edge pedestal in the ion temperature profiles. As demonstrated in Fig. 2, at the transition regime from ion root to electron root, the ion temperature increases associated with the transition as the electron density is decreased, while there is no 

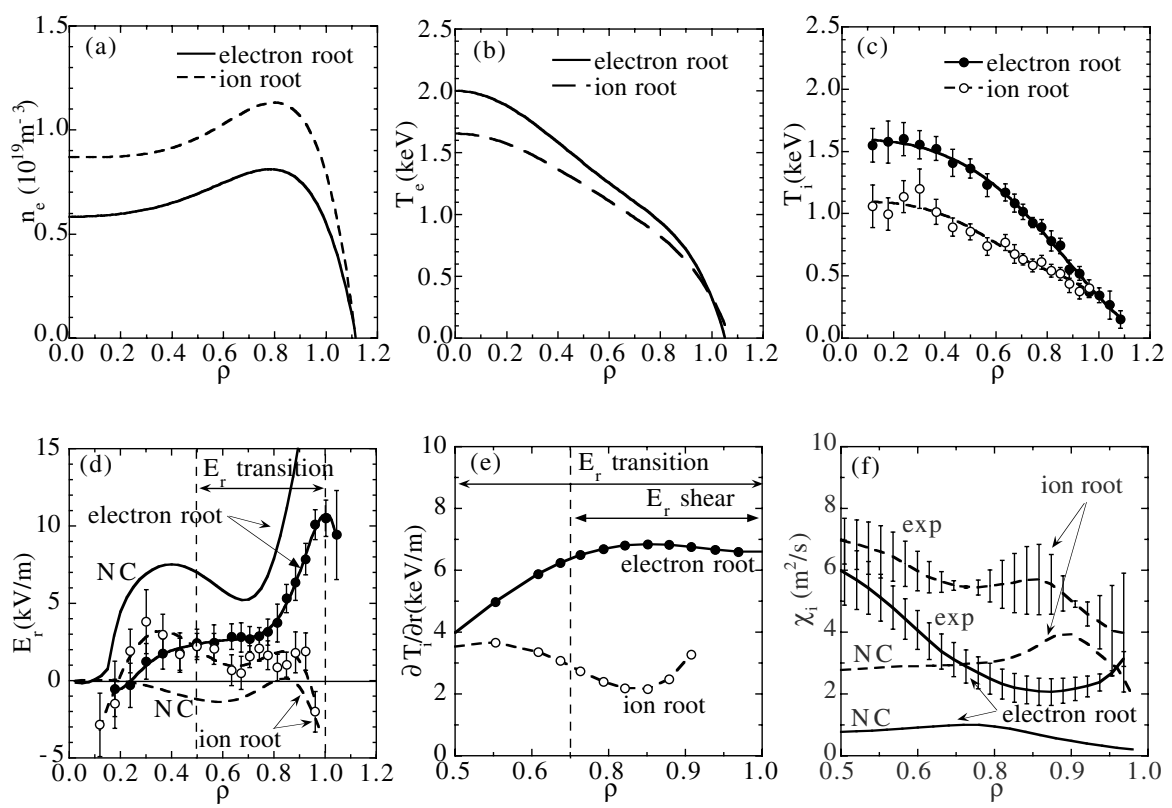

FIG. 2. Radial profiles of (a) electron density, (b) electron temperature, (c) ion temperature, (d) radial electric field, (e) ion temperature gradient, and (f) ion thermal diffusivity for the electron root (closed circles) and ion root (open circles) plasmas. The dashed lines in (d) and (f) are neoclassical predictions.

significant change of electron temperature for the plasma with $R_{\mathrm{ax}}=3.75 \mathrm{~m}$. On the other hand, the ion temperature slightly decreases as the electron density is decreased for the plasma with $R_{\mathrm{ax}}=3.6 \mathrm{~m}$, regardless of the transition. As shown in Fig. 2(d), the radial electric field near the edge changes from a negative value (ion root) to positive values (electron root) as the electron density is decreased. The measured radial electric field is compared with that predicted by neoclassical theory. The radial electric field $E_{r}$ is calculated with the balance of neoclassical ion flux and electron flux using the measured electron density, electron temperature, and ion temperature profiles [15]. The $E_{r}$ calculated with neoclassical theory in the electron root becomes larger towards the plasma edge as shown in Fig. 2(d), which shows qualitative agreement with the measured $E_{r}$.

The increase of the ion temperature gradient is most significant at $\rho>0.6$, where the transition from ion root to electron root occurs. The ion temperature gradient increases towards the plasma edge up to $7 \mathrm{keV} / \mathrm{m}$ in the electron root, while it is only $2 \mathrm{keV} / \mathrm{m}$ in the ion root. It should be noted that the temperature gradient in the ion root and electron root in LHD is comparable to that in the $L$ mode $(2-3 \mathrm{keV} / \mathrm{m})$ and in the $H$-mode discharges $(5-25 \mathrm{keV} / \mathrm{m})$ in D-IIID, respectively [16]. As shown in Fig. 2(e), the ion temperature gradient multiplied by the electron density in the electron root is twice that in the ion root. This suggests the improvement of ion transport in the electron root. The transport analysis shows that the ion diffusivity at $\rho=0.85$ is reduced from 5.5 to $2 \mathrm{~m}^{2} / \mathrm{s}$ (more than a factor of 2) associated with the transition from ion root to electron root as shown in Fig. 3(f). The beam power deposition profiles are calculated with a three-dimensional Monte Carlo simulation code [17] including orbit loss and charge exchange loss. The reduction of ion thermal diffusivity predicted by neoclassical theory is much larger than that observed. This fact suggests that the anomalous transport is of the order of $2 \mathrm{~m}^{2} / \mathrm{s}$ at the plasma edge. The gradient of radial electric field $\left(E_{r}\right.$ shear $)$ is $120 \mathrm{kV} / \mathrm{m}^{2}$ at $\rho=0.9$ in the electron root, and it is not large enough to suppress the anomalous transport. The $E_{r}$ shear required to suppress the fluctuations and to improve electron transport is $350 \mathrm{kV} / \mathrm{m}^{2}$ in a compact helical system [10]. Therefore, larger $E_{r}$ shear is expected to be necessary to suppress the fluctuations and improve anomalous transport in the electron root plasma in LHD.

Figure 3 shows the relation between ion thermal diffusivity and radial electric field near the plasma edge $(\rho \sim$ $0.9)$ at the density of the transition regime $\left[n_{e}(0.9)=\right.$ $(0.4-1.2) \times 10^{19} \mathrm{~m}^{-3}$ for $R_{\mathrm{ax}}=3.75 \mathrm{~m}$ and $n_{e}(0.9)=$ $(0.2-0.5) \times 10^{19} \mathrm{~m}^{-3}$ for $\left.R_{\mathrm{ax}}=3.6 \mathrm{~m}\right]$. The neoclassical ion thermal diffusivity for the given radial electric field is also calculated. The neoclassical ion thermal diffusivity becomes maximum at zero radial electric field and decreases as the radial electric field increases both for negative and positive $E_{r}$. This is in contrast with the disappearance of the peak of the ion thermal diffusivity at zero radial electric field in the plasma with $R_{\mathrm{ax}}=3.6 \mathrm{~m}$. Since (i) the $\mathbf{E} \times \mathbf{B}$ rotation is in the same direction as the $\operatorname{grad}-B$ drift so as to reduce the radial deviation of banana orbit and (ii) the absolute values of radial electric field in the electron root are much larger than those in the ion root, a larger reduction of ion thermal diffusivity is expected from neoclassical theory. In experiment, in the density regime of transition from ion to electron root, the absolute values of the radial electric field in the ion root are only 

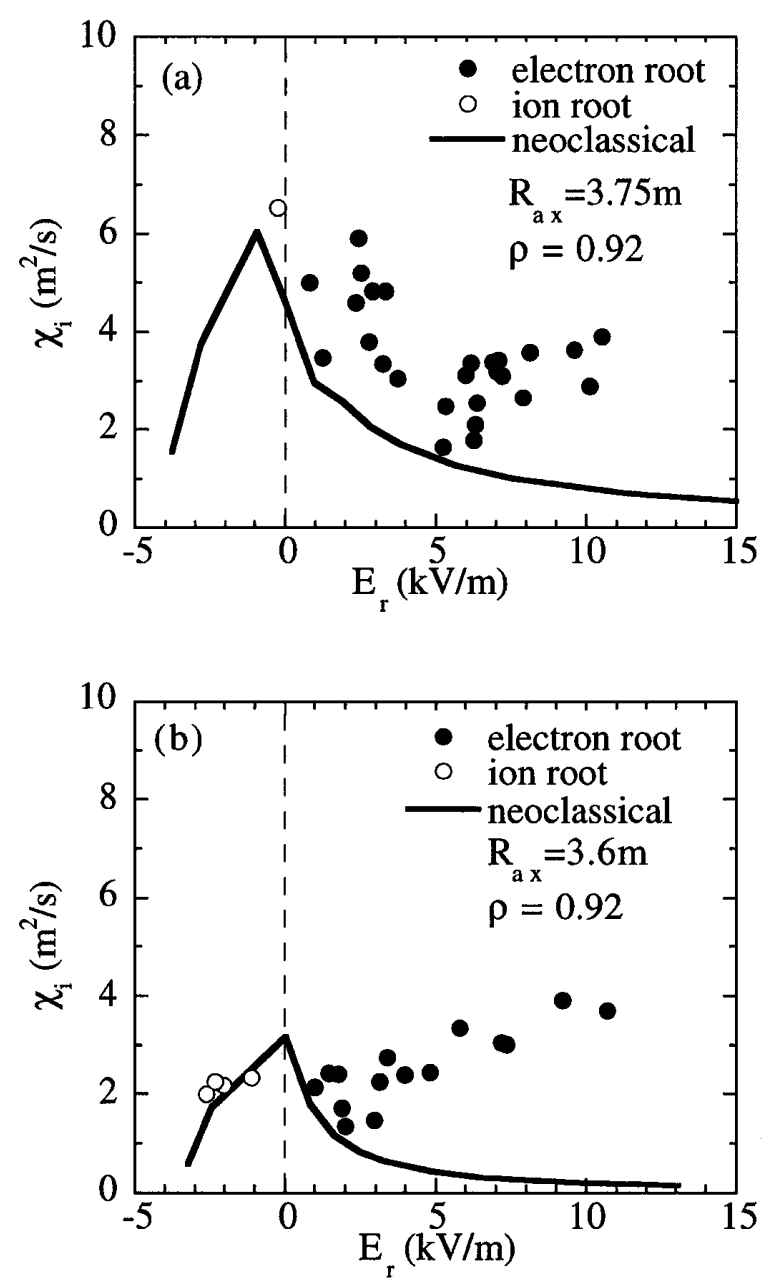

FIG. 3. Ion thermal diffusivity as a function of radial electric field at $\rho=0.92$ for the plasmas with (a) standard configuration $\left(R_{\mathrm{ax}}=3.75 \mathrm{~m}\right)$ and (b) inward shifted configuration $\left(R_{\mathrm{ax}}=3.6 \mathrm{~m}\right)$.

$1-3 \mathrm{kV} / \mathrm{m}$, while they increase sharply up to $10 \mathrm{kV} / \mathrm{m}$ in the electron root.

The reduction of ion thermal diffusivity sharply decreases as the positive electric field increases after the transition from ion root to electron root in the plasma with $R_{\mathrm{ax}}=3.75 \mathrm{~m}$, which qualitatively agrees with the prediction from neoclassical theory. Once the neoclassical ion diffusivity decreases below the anomalous levels $\left(\sim 2 \mathrm{~m}^{2} / \mathrm{s}\right)$, there is no reduction of ion thermal diffusivity observed. The reduction of ion thermal diffusivity in the electron root observed in LHD standard configuration supports the validity of the concept for the stellarator configuration, where the helical ripple loss is suppressed by the radial electric field even in the low collisionality regime. Moreover, the disappearance of the peak of the ion thermal diffusivity at zero radial electric field in the plasma with $R_{\mathrm{ax}}=3.6 \mathrm{~m}$ clearly shows the significant reduction of helical ripple loss due to the inward shift of magnetic axis which is predicted from neoclassical theory. The anomalous transport near the edge is comparable or even smaller than that observed in the $L$-mode plas- mas $\left[\chi_{i}=(3-10) \mathrm{m}^{2} / \mathrm{s}\right]$ in tokamaks [18], although the anomalous transport in the core region is comparable to that in tokamaks [19]. In order to suppress the anomalous transport, the $E_{r}$ shear (not just $E_{r}$ ) should be produced similar to that in the transport barrier in tokamak plasmas.

In conclusion, the two crucial neoclassical features of "ion" heat transport are experimentally confirmed for the first time in the NBI heated plasmas in a Heliotron. The ion thermal diffusivity is found experimentally to be significantly reduced by (1) the transition from small negative $E_{r}$ (ion root) to large positive $E_{r}$ (electron root) or (2) the optimization of magnetic field configuration (inward shift of magnetic axis), which are predicted by the neoclassical theory in stellarator plasmas.

The authors thank the technical support of the deviceengineering group for LHD. Useful discussions with Professor S.-I. Itoh of Kyushu University are also appreciated.

*Present address: High temperature Plasma Center, University of Tokyo, Tokyo, 113-8656, Japan.

†Present address: JET, Abindon, Oxforshire, United Kingdom.

\#Department of Fusion Science, School of Mathematical and Physical Science, Graduate University for Advanced Studies, Hayama, 240-0193, Japan.

${ }^{\S}$ Department of Energy Engineering and Science, Nagoya University, 464-8603, Japan.

[1] H. P. Furth and M. N. Rosenbluth, in Proceedings of the 3rd International Conference on Plasma Physics and Controlled Nuclear Fusion Research, 1968 (IAEA, Vienna, 1969), Vol. 1, p. 821.

[2] S. P. Hirshman and D. J. Sigmar, Nucl. Fusion 21, 1079 (1981).

[3] Y. Ogawa et al., Nucl. Fusion 32, 119 (1992).

[4] H. E. Mynick, T. K. Chu, and A. Boozer, Phys. Rev. Lett. 48, 322 (1982).

[5] H. Sanuki, J. Todoroki, and T. Kamimura, Phys. Fluids B 2, 2155 (1990).

[6] K. Itoh, H. Sanuki, J. Todoroki, T. Kamimura, S.-I. Itoh, A. Fukuyama, and K. Hanatani, Phys. Fluids B 3, 1294 (1991).

[7] H. Idei et al., Phys. Rev. Lett. 71, 2220 (1993).

[8] A. Fujisawa et al., Phys. Rev. Lett. 81, 2256 (1998).

[9] J. Baldzuhm et al., Plasma Phys. Controlled Fusion 40, 967 (1998).

[10] A. Fujisawa et al., Phys. Rev. Lett. 82, 2669 (1999).

[11] H. Maassberg et al., Phys. Plasmas 7, 295 (2000).

[12] M. Fujiwara et al., Nucl. Fusion 40, 1157 (2000).

[13] K. Ida, S. Kado, and Y. Liang, Rev. Sci. Instrum. 71, 2360 (2000).

[14] N. Ohyabu et al., Phys. Rev. Lett. 84, 103 (2000).

[15] K. Yamazaki and T. Amano, Nucl. Fusion 32, 633 (1992).

[16] R. D. Deranian, R. J. Groebner, and D. T. Pham, Phys. Plasmas 7, 1235 (2000).

[17] S. Murakami, N. Nakajimja, and M. Okamoto, Trans. Fusion Technol. 27, 256 (1995).

[18] Y. Kamada, Plasma Phys. Controlled Fusion 42, A65 (2000).

[19] H. Yamada et al., Phys. Rev. Lett. 84, 1216 (2000). 